\title{
A Method to Obtain the Integrated Contour Image in Colour Scenes
}

\author{
Xavier Cufí, Alícia Casals ${ }^{* *}$, Joan Batlle ${ }^{*}$ \\ *University of Girona \\ Institute of Informatics and Applications \\ Av. Lluís Santaló s/n, 17003 Girona, Spain \\ ${ }^{* *}$ Polytechnical University of Catalonia \\ Dep. of Automatic Control and Computer Engineering \\ C/ Pau Gargallo5, 08028 Barcelona, Spain
}

\begin{abstract}
This paper describes a method to achieve the most relevant contours of an image.

The presented method proposes to integrate the information of the local contours from chromatic components such as $H, S$ and $I$, taking into account the criteria of coherence of the local contour orientation values obtained from each of these components. The process is based on parametrizing pixel by pixel the local contours (magnitude and orientation values) from the $H, S$ and I images. This process is carried out individually for each chromatic component. If a criteria of dispersion of the obtained orientation values is high, this chromatic component will lose relevance. A final processing integrates the extracted contours of the three chromatic components, generating the so-called integrated contours image.
\end{abstract}

\section{Introduction}

It is known that colour provides an additional information source with respect to monochromatic images, which supposes a significant increase in the robustness of the computational processes applied to images. Therefore, it is logical to think of the use of the different chromatic components in the process of contour extraction. The images to be processed must obviously contain relevant chromatic information.

Some researchers have been working on the extraction of contour information from colour images. Nevatia [1] suggests three possibilities to detect the contours of a colour image: (a) to define a determined metrics in a tridimensional colour space, (b) to perform an integration of the obtained contours on every one of the chromatic components, and (c) to assume that the contours obtained from the three chromatic components are independent.

This author works with colour images in the $\mathrm{Y}$, T1, T2 space, which is similar to the RGB space, and uses an extension of the Hueckel operator to detect the contours on which coherence restrictions in the orientation values obtained in each component, are applied.

More recently, Carron and Lambert [2] determined the gradient of colour images from the HSV chromatic components. These authors also propose information integration mechanisms of the local contours obtained from each chromatic component. This integration can be performed considering either that the contours of all the components provide the same information, or considering that data from the hue component provides the most relevant information of the colour image contours.

In the work presented in this paper, the original colour image is transformed to the colour space HSI. This transformation is by many authors considered as the most appropriate one for the segmentation of colour images [2], [3].

Since the representation in the RGB space is not related to the concept and the subjective perception of the colour realized by human beings, we have used the HSI colour model in the results here presented. However, the system could use other colour models perhaps more adequate according to the work environment.

\section{Obtention of Chromatic Local Contours}

From the hue, intensity and saturation images of a scene, we obtain the local edges (magnitude and orientation) using a method based on compass gradient masks [4]. They are represented as $\mathrm{C}^{\mathrm{H}}\left(\mathrm{M}^{\mathrm{H}}, \mathrm{O}^{\mathrm{H}}\right)$, $\mathrm{C}^{\mathrm{l}}\left(\mathrm{M}^{\mathrm{I}}, \mathrm{O}^{\mathrm{l}}\right), \mathrm{C}^{\mathrm{S}}\left(\mathrm{M}^{\mathrm{S}}, \mathrm{O}^{\mathrm{S}}\right)$ respectively. The obtained 
orientation values $\left(\mathrm{O}^{\mathrm{i}}\right)$ are coded as a compass: $\mathrm{N}, \mathrm{NE}$, E, SE, S, SW, W, NW.

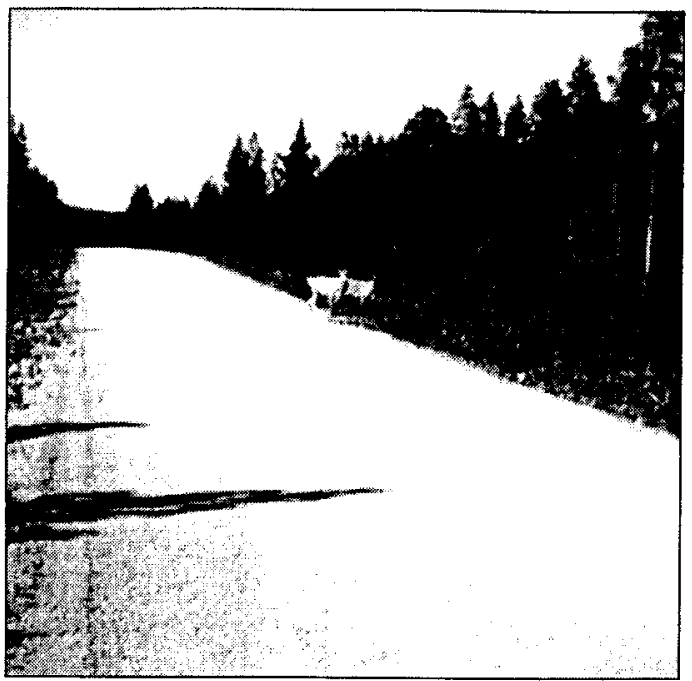

Fig 1: Original Image (intensity component)

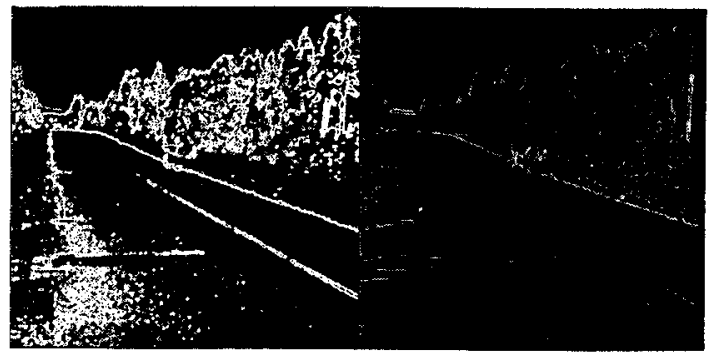

(a)

(b)

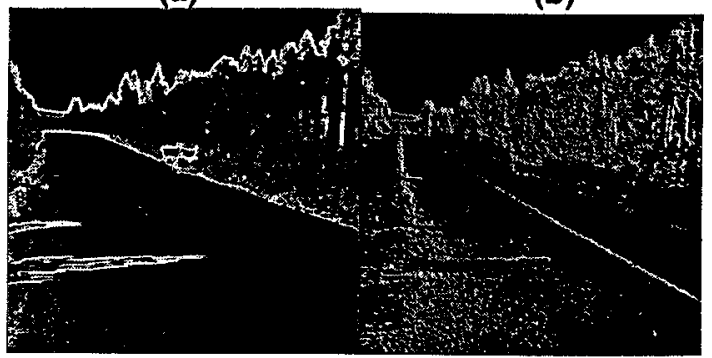

(c)

(d)

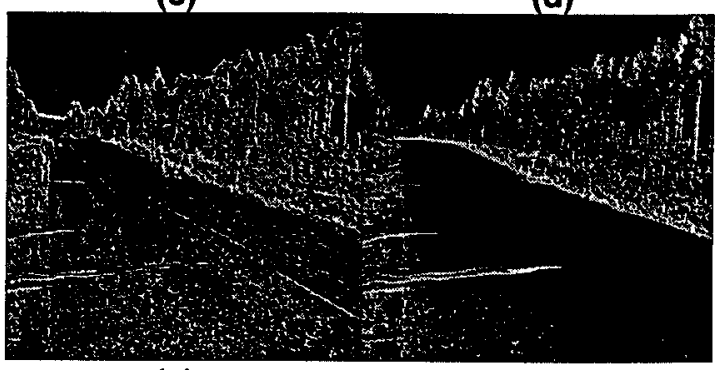

(e)

(f)

Fig. 2. Magnitude and orientation of local contours of $\mathrm{H}, \mathrm{S}$ and $\mathrm{I}$. (a) $\mathbf{M}^{\mathrm{H}}$, (b) $M^{\mathrm{s}}$, (c) $M^{\mathrm{j}}$, (d) $\mathrm{O}^{\mathrm{H}},(\mathrm{e}) \mathrm{O}^{\mathrm{s}},(f) \mathrm{O}^{\prime}$.
Figure 2 shows the magnitude and orientation of the local contours obtained from the $\mathrm{H}, \mathrm{S}$ and I components of the scene shown in fig. 1 .

The integration of the previously obtained edge images enables the selective extraction of the most relevant contour information from each of them. A new integrated contours image is then generated, considering:

$$
f(C)=f\left(C^{H}, C^{\prime}, C^{S}\right)
$$

In Huang's work [5] colour image contours are achieved by integrating the information of the images of the local gradients obtained from the $R, G$ and $B$ components. The integration function used by these authors corresponds to the maximum value of the gradient magnitude of each component, determined on each pixel of the image, without considering any additional local information.

\section{Generation of Integrated Contour Image}

In our work we define an improved integration function that takes into consideration some local information in a neigbourdhood of the pixels belonging to the chromatic components contours. The integration function is defined as follows:

$$
f(C)=\max \left(\gamma_{H}\left(O^{H}\right) M^{H}, \gamma_{I}\left(O^{l}\right) M^{l}, \gamma_{S}\left(O^{S}\right) M^{S}\right)
$$

The weighting factors $\gamma_{i}\left(\mathrm{O}^{i}\right)$, take into account the gradient magnitude and orientation smoothness within a nxn region surrounding the pixel considered. Such factors are represented in vectorial form. For each region of size nxn (in the chromatic component i), it is possible to represent the number of pixels which present a determinated value of the local orientation $k$, in form of the module of a vector $V(i, k)$.

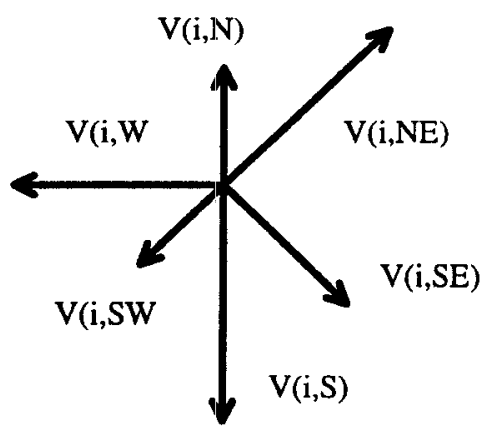

Fig. 3. Vectorial distribution of local orientation values, in a region of $n \times n$ pixels (for the chromatic component i).

The global behaviour of the orientation in this region, is determinated by the module of the vector 
resulting from the vectorial addition of all $V(i, k)$ contributions in that region. Consequently,

$$
\gamma_{i}\left(O_{i}\right)=\frac{1}{2 n} \bmod \left(\sum_{k} V(i, k)\right)
$$

where $V(i, k)$ are the vectors associated with the values of local orientation in the neigbourhhod $(k=\{N, N E, E$, SE, S, SW, W, NW $\}$ ). The index i corresponds with any of the chromatic compunents $\{\mathrm{H}, \mathrm{S}, \mathrm{I}\}$.

Therefore, one can consider a region which presents an orientation vector beings zero in case there isn't any contour information (the local orientation vectors $V(i, k)$ have each one, a small value of module) or in case the information value, in this region is very dispersed (the vectorial addition of all the local orientation values, results in a vector $\mathrm{O}^{i}$ with a small module).

If the parameter $\gamma_{\mathrm{i}}\left(\mathrm{O}^{\mathrm{i}}\right)$ approaches zero, this neighbourhood is considered to have no linearity. This implies a lack of coherence in the orientation values and, therefore, the products $\gamma_{\mathrm{i}}\left(\mathrm{O}^{\mathrm{i}}\right) \mathrm{M}^{\mathrm{i}}$ become insignificant.

The vector $\mathrm{O}^{\mathrm{i}}$ may represent the orientation of the region in case the value of the module is significant enough or, in other words, when the pixels of the region present some values of local orientation dominant to the others and consequently confirms the existence of coherence in the orientation values. This situation normally occurs in case there exists a well defined local contour.

\section{Results}

In Figure 4a, we show a zone (in the intensity component) where the value of the factor $\gamma_{i}$ is high. In this case, the local contours obtained from the intensity component are relevant. Figure $4 \mathrm{~b}$ shows a zone on the intensity component, where the value of the factor $\gamma_{1}$ is small since the orientation value of the contours in this zone is very scattered. In such case, the intensity component may result of poor relevance. Figures $5 \mathrm{a}$ and $5 b$ show respectively the value distribution of the local orientation shown in figures $4 a$ and $4 b$.

This methodology has been tested for very different kind of images. In the following, we show an application exemple for the natural scene of fig. 1 .

Figure 6 shows the integrated contours image, obtained using the abovementioned method.

The integrated contour images obtained constitute a first step in the obtaining the most significant perceptive contours of a natural image, which will facilitate a further segmentation process [6].

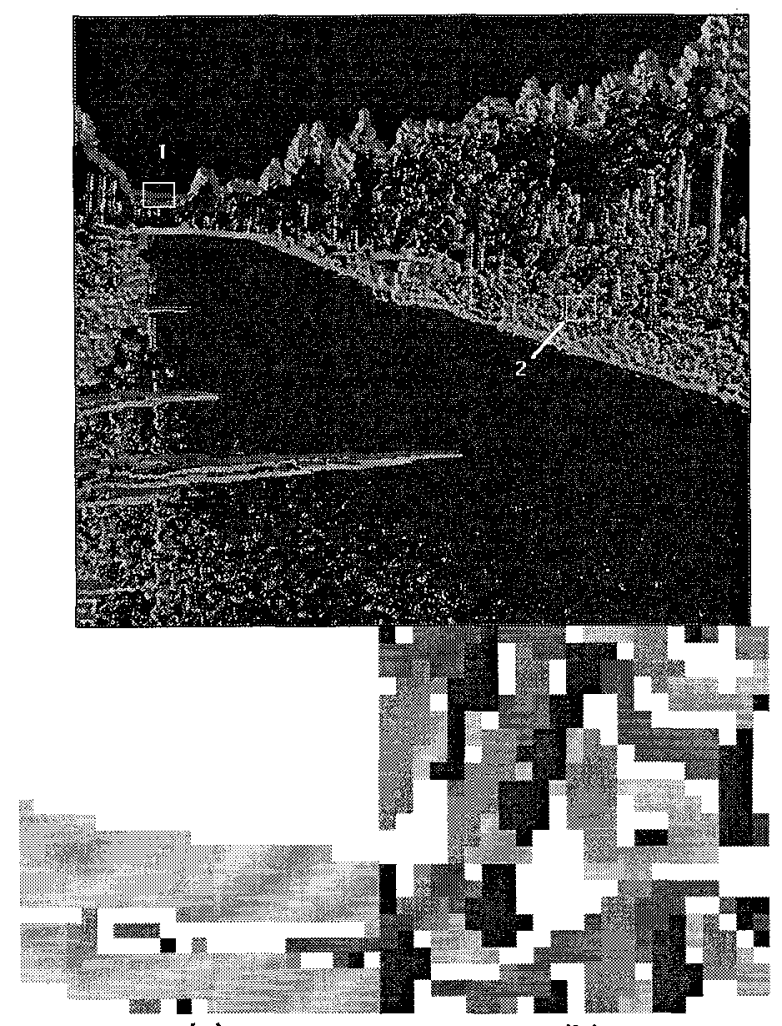

(a)

(b)

Fig. 4. (a) subimage 1, from the intensity component (a zone with a relevant contour). (b) subimage 2, from the intensity component (scattered values of local contour information).
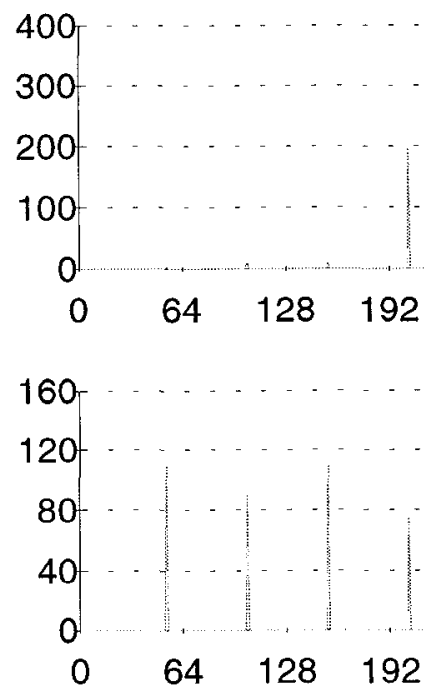

Fig. 5(a) Distribution of local orientations for subimage $1\left(\gamma_{1}\left(O^{\prime}\right)=0.8\right)$. 5(b) Distribution of local orientations for subimage $2\left(\gamma_{1}\left(O^{\prime}\right)=0.1\right)$. 


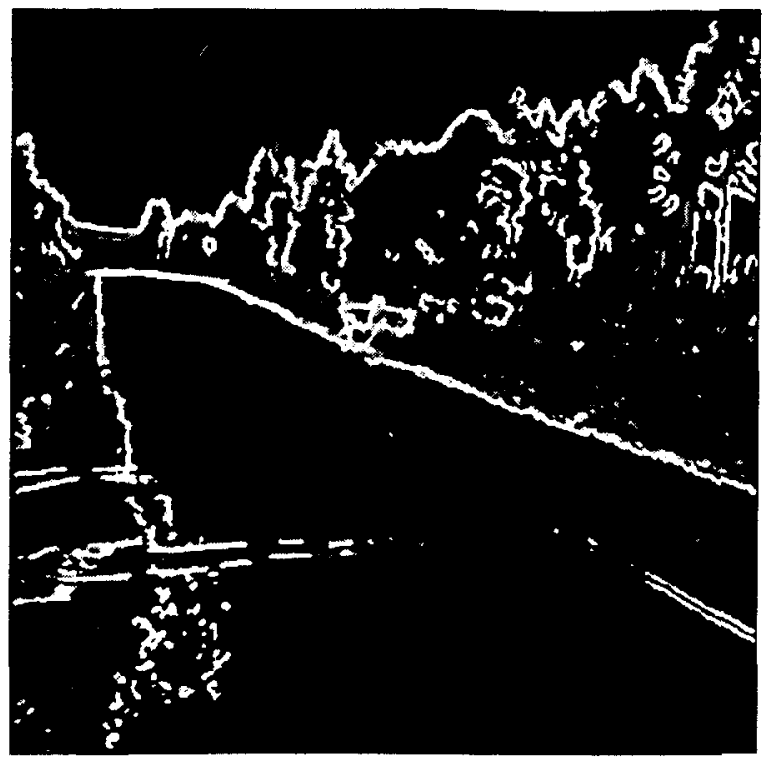

Fig. 6. Integrated contour image obtained, using a $5 \times 5$ pixels neigbourhood.

\section{References}

[1] R. Nevatia. "A Color Edge Detector and Its Use in Scene Segmentation". IEEE Trans. on SMC, Vol. $7 \mathrm{~N}^{\circ} 11.1977$.

[2] T. Carron, P. Lambert. "Color Edge Detector using jointly Hue, Saturation and Intensity". International Conference on Image Processing. 1994.

[3] P.W.M. Tsang, W.H. Tsang. "Edge detection on Object Color". Proc. on International Conference on Image Processing (IEEE). 1996

[4] G.S. Robinson. "Color Edge Detection". Optical Engineering, Vol. 16, No 5. 1977.

[5] Q. Huang, B. Doom, D. Steele, J. Ashley, W. Niblack. "Foreground/Background Segmentation of Color Images by Integration of Multiple Cues". Proc. on the International Conference on Image Processing (IEEE). 1995.

[6] X. Cufí, A. Casals "A Criterion for Circumscribed Contours Determination for Image Segmentation". Proc. on IAPR Mą̧hine Vision Applications. 1996. 